\title{
The influence of the quality of the solvent on the properties of a polymer
}

\section{A thermodynamic model and molecular dynamics calculations}

\author{
by B. SMIT and K. R. COX $\dagger$ \\ Koninklijke/Shell Laboratorium Amsterdam (Shell Research B.V.), \\ Badhuisweg 3, $1031 \mathrm{CM}$ Amsterdam, The Netherlands \\ and J. P. J. MICHELS \\ Van der Waals Laboratorium, Universiteit van Amsterdam, \\ P.O. Box 20216, 1000 HE Amsterdam, The Netherlands
}

(Received 8 June 1988; accepted 18 August 1988)

Computer simulations of a polymer immersed in a solvent are presented. The influence of the quality of the solvent on the static and dynamic properties of a polymer has been studied. The quality of the solvent is modelled by varying the depth $\left(\varepsilon_{\mathrm{mp}}\right)$ of the Lennard-Jones potential, which describes the interactions of the polymer segments with the monomers.

Starting from an isolated chain $\left(\varepsilon_{\mathrm{mp}}=0\right)$, the introduction of a small polymer solvent interaction gives rise to a large decrease of the radius of gyration. At very low values of $\varepsilon_{\mathrm{mp}}$ the interactions between the polymer segments and monomers is mainly repulsive. Such an unattractive solvent causes the polymer chain to collapse. A further increase of $\varepsilon_{\mathrm{mp}}$ causes the radius of gyration to increase again. A simple thermodynamic model, the 'polymer in a sack model', is introduced to describe this behaviour of the radius of gyration quantitatively. In this model we introduce some assumptions for handling the chain statistic of the polymer. With these assumptions the calculations reduce to a classical phase equilibrium problem. It is also demonstrated that the quality of the solvent can be used as a coupling parameter in Kirkwood's coupling parameter formalism. With this formalism we were able to calculate differences in free energy.

\section{Introduction}

In many technical applications systems are encountered in which large molecules are immersed in a solvent of small molecules. Therefore it is of practical importance to have a detailed understanding of the influence of the solvent on the properties of these large molecules. Computer simulations of simple model systems may provide some insight into the behaviour of these systems.

Over the past few years several authors [1-13] have studied the influence of the solvent on the properties of a polymer. The extent to which the static properties of a polymer (i.e. radius of gyration or end-to-end distance) are influenced by the solvent is still a matter of controversy. Khalatur et al. [10] observed that the behaviour of a polymer immersed in a solvent containing monomers is mainly determined by intrachain rather than solvent effects. In contrast to these observations Smit et al. [11] found a significant influence of the density of the solvent on the static properties of a star polymer.

$\dagger$ Present address: Shell Development Company, Westhollow Research Center, P.O. Box 1380, Houston, Texas 7751-1380. 
All these studies related to a polymer in a solvent containing its own segments. It is well known that the shape of a polymer is influenced by the chemical nature of the solvent. In a recent study Smit et al. [13] reported a molecular dynamics simulation in which they studied the influence of the chemical nature of the solvent on the radius of gyration of the polymer. The chemical nature or quality of the solvent was modelled by varying the $\varepsilon_{\mathrm{mp}}$ of the Lennard-Jones potential, which describes the interaction of the polymer segments with the solvent. They observed that compared to an isolated chain $\left(\varepsilon_{\mathrm{mp}}=0\right)$, the introduction of a very small interaction between the polymer segments and the monomers of the solvent gives rise to a decrease of the radius of gyration of 25 per cent. As the interaction is further increased the radius of gyration starts to increase again.

In order to gain a better understanding of this unexpected behaviour of the radius of gyration of the polymer, we have developed a simple model, " polymer in a sack model', which will be presented here. This model is based on a modification of the van der Waals equation of state. Despite the simplicity of this model a semiquantitative description of the data of Smit et al. [13] was obtained. In order to test this model we have performed some additional simulations.

For estimating the phase behaviour of these systems it is important to calculate the free energy. Quantities related to the free energy are difficult to obtain directly from computer simulations [14]. In this paper we will demonstrate that the quality of the solvent can be used as a coupling parameter in Kirkwoods' coupling parameter formalism. With this formalism it is possible to calculate free energy differences.

We have divided this paper in three parts. In the first part we describe the computer simulations, in the second we present the results of these simulations, and in the third we present the 'polymer in a sack model' model which describes the behaviour of the radius of gyration.

\section{Model and computational details}

In the simulations presented in this paper we have studied a linear polymer having eight segments in a solvent containing 492 particles. This model is the same as we have used in a previous study [13], and the main features are outlined in this section.

The interaction potential consists of two parts: a shifted Lennard-Jones potential which operates between all 500 particles and a harmonic potential which links the eight particles into a linear polymer [13].

The shifted Lennard-Jones potential is given by

$$
U_{\mathrm{LJ}}\left(r_{i j}\right)= \begin{cases}4 \varepsilon_{i j}\left[\left(\sigma / r_{i j}\right)^{12}-\left(\sigma / r_{i j}\right)^{6}\right]-U_{\mathrm{LJ}}\left(r^{\mathrm{c}}\right), & r_{i j} \leqslant r^{\mathrm{c}}, \\ 0, & r_{i j}>r^{\mathrm{c}},\end{cases}
$$

with $r^{\mathrm{c}}=2 \cdot 5 \sigma$. In some simulations we have used $r^{\circ}=2^{1 / 6} \sigma$.

In most simulations it is assumed that the polymer is immersed in a solvent of its own segments. This implies that

$$
\begin{aligned}
\varepsilon_{\mathrm{mm}} & =\varepsilon_{\mathrm{pp}}=\varepsilon^{0} \\
\varepsilon_{\mathrm{mp}} & =\left(\varepsilon_{\mathrm{mm}} \varepsilon_{\mathrm{pp}}\right)^{1 / 2}=\varepsilon^{0} \\
\sigma_{\mathrm{mp}} & =\sigma_{\mathrm{mm}}=\sigma_{\mathrm{pp}}=\sigma .
\end{aligned}
$$




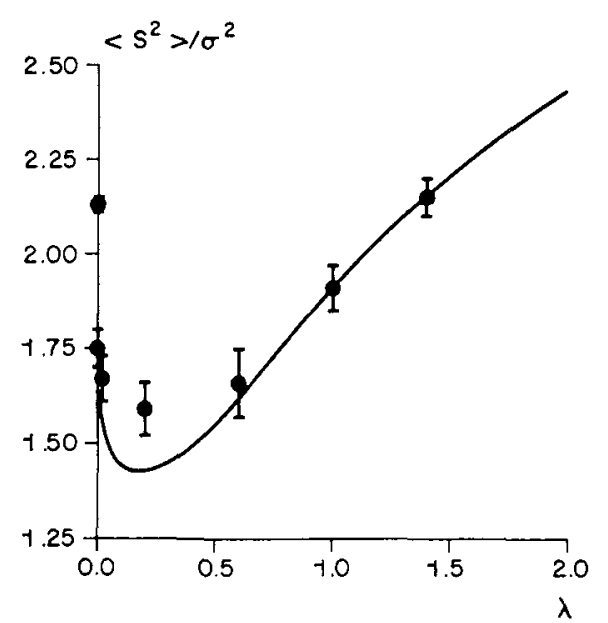

Figure 1. The radius of gyration $(S)$ as a function of the quality of the solvent $\lambda$ with $r^{c}=2 \cdot 5 \sigma$. The dots are obtained from computer simulations and the line is calculated with the polymer in a sack model.

The subscript $\mathrm{mm}$ indicates monomer-monomer interactions, $\mathrm{pp}$ denotes polymer segment-polymer segment interactions and mp denotes monomer-polymer segment interactions.

In order to study the influence of the quality of the solvent on the properties of the polymer we have introduced a parameter $\lambda$ in the expression for the interaction parameter of the polymer segment-monomer:

$$
\varepsilon_{\mathrm{mp}}=\lambda\left(\varepsilon_{\mathrm{mm}} \varepsilon_{\mathrm{pp}}\right)^{1 / 2}=\lambda \varepsilon^{0} .
$$

When $\lambda<1$, the depth of the Lennard-Jones potential is less than the reference $\left(\varepsilon^{0}\right)$, so the quality of the solvent is poorer than this reference. When $\lambda>1$ the quality of the solvent is better than the reference.

Although we have tried to incorporate some of the characteristics of real polymer systems, this model of a polymer and the concept of quality of the solvent as a model for the chemical structure are, of course, far from an accurate description of real polymer systems.

In the simulations, periodic boundary conditions are imposed in all three directions. The equations of motion are solved with Verlet's integration scheme [15] with a time step $\Delta t^{*}=0.007 t / \sigma(m / \varepsilon)^{1 / 2}$. All the simulations have been performed at a reduced temperature $T^{*}=2 \cdot 0 k T / \varepsilon$ and a reduced density $\rho^{*}=0.6 \rho \sigma^{3}$. Estimates of the standard deviation are obtained by dividing the samples into five groups and calculating the group averages.

\section{Results of the computer simulations}

\subsection{Shape of the polymer}

The shape of a polymer is often characterized by the end-to-end distance $(R)$, the radius of gyration $(S)$, and the three principal orthogonal components of the inertia tensor; $0 \leqslant\left\langle l_{1}^{2}\right\rangle \leqslant\left\langle l_{2}^{2}\right\rangle \leqslant\left\langle l_{3}^{2}\right\rangle[11,13]$.

The values of the radius of gyration and end-to-end distance for several values of $\lambda$ are presented in figures 1,2 and table 1 . The results given in figure 1 and table $1(a)$ 


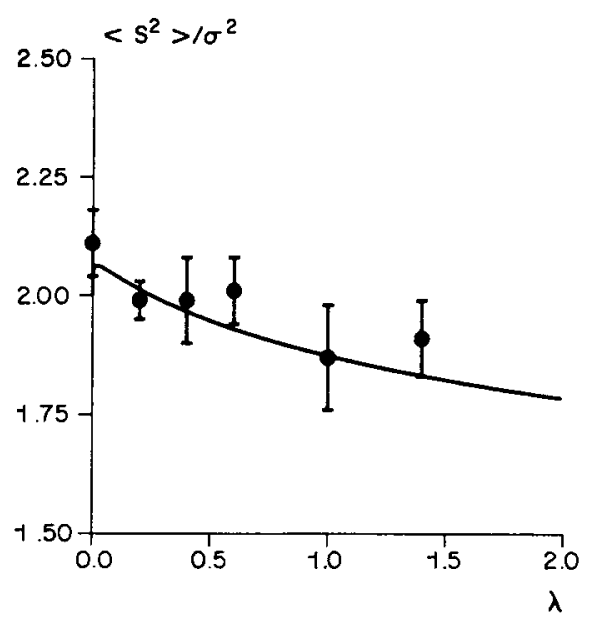

Figure 2. The radius of gyration $(S)$ as a function of the quality of the solvent $\lambda$ with $r^{c}=2^{1 / 6} \sigma$. The dots are obtained from computer simulations and the line is calculated with the polymer in a sack model.

concern simulations in which the Lennard-Jones potential has been truncated at $r^{\mathfrak{c}}=2 \cdot 5 \sigma$, so both the repulsive part and the attractive part are taken into account. The results given in figure 2 and table $1(b)$ concern simulations in which only the repulsive part of the Lennard-Jones potential is taken into account by truncating the potential at $r^{\mathrm{c}}=2^{1 / 6} \sigma$.

The eigenvalues of the inertia tensor for the simulations with $r^{c}=2.5 \sigma$ are presented in table 2 . These results show that the polymer stretches at high and very low values of $\lambda$.

Table 1. Influence of the quality of the solvent on the radius of gyration of a polymer. The reduced temperature for each simulation was $T^{*}=2.0$ and the reduced density was $\rho^{*}=0.6$. In (a) the potential was truncated at $r^{\mathrm{c}}=2.5 \sigma$ and in $(b)$ at $r^{\mathrm{c}}=2^{1 / 6} \sigma$.

(a)

\begin{tabular}{clcr}
\hline No. & \multicolumn{1}{c}{$\lambda$} & \multicolumn{1}{c}{$\left\langle S^{2}\right\rangle$} & \multicolumn{1}{c}{$\left\langle R^{2}\right\rangle$} \\
\hline 1 & 0.00 & $2.13 \pm 0.02$ & $14.03 \pm 0.42$ \\
2 & 0.0002 & $1.75 \pm 0.05$ & $10.16 \pm 0.57$ \\
3 & 0.02 & $1.67 \pm 0.06$ & $9.13 \pm 0.74$ \\
4 & 0.20 & $1.59 \pm 0.07$ & $8.58 \pm 0.74$ \\
5 & 0.60 & $1.66 \pm 0.09$ & $9.47 \pm 0.94$ \\
6 & 1.00 & $1.91 \pm 0.06$ & $11.62 \pm 0.68$ \\
7 & 1.40 & $2.15 \pm 0.05$ & $13.24 \pm 0.82$ \\
\hline \multicolumn{4}{c}{$(b)$} \\
\hline 2 & 0.0002 & $2.13 \pm 0.06$ & $13.75 \pm 0.72$ \\
3 & 0.02 & $2.13 \pm 0.09$ & $13.43 \pm 1.27$ \\
4 & 0.20 & $1.99 \pm 0.04$ & $12.25 \pm 0.42$ \\
5 & 0.60 & $1.99 \pm 0.09$ & $12.45 \pm 1.04$ \\
6 & 1.00 & $1.87 \pm 0.11$ & $10.72 \pm 1.09$ \\
7 & 1.40 & $1.91 \pm 0.08$ & $11.39 \pm 0.08$ \\
\hline
\end{tabular}


Table 2. Eigenvalues of the inertia tensor as a function of the quality of the solvent (the potential was truncated at $R^{\mathrm{c}}=2 \cdot 5 \sigma$ ).

\begin{tabular}{clccc}
\hline No. & \multicolumn{1}{c}{$\lambda$} & \multicolumn{1}{c}{$\left\langle l_{1}^{2}\right\rangle$} & $\left\langle l_{2}^{2}\right\rangle$ & $\left\langle l_{3}^{2}\right\rangle$ \\
\hline 1 & 0.0 & $0.042 \pm 0.002$ & $0.131 \pm 0.010$ & $0.826 \pm 0.010$ \\
2 & 0.0002 & $0.058 \pm 0.004$ & $0.176 \pm 0.006$ & $0.766 \pm 0.026$ \\
3 & 0.02 & $0.059 \pm 0.002$ & $0.181 \pm 0.005$ & $0.759 \pm 0.038$ \\
4 & 0.20 & $0.064 \pm 0.008$ & $0.190 \pm 0.020$ & $0.746 \pm 0.097$ \\
5 & 0.60 & $0.062 \pm 0.004$ & $0.181 \pm 0.016$ & $0.755 \pm 0.069$ \\
6 & 1.00 & $0.047 \pm 0.002$ & $0.157 \pm 0.011$ & $0.796 \pm 0.036$ \\
7 & 1.40 & $0.043 \pm 0.005$ & $0.144 \pm 0.006$ & $0.813 \pm 0.032$ \\
\hline
\end{tabular}

Table 3. Centre-of-mass diffusion coefficient as a function of the quality of the solvent $(\lambda)$ ( $D^{\text {ar }}$ diffusion coefficient for the simulations with $r^{c}=2 \cdot 5 \sigma$ and $D^{r}$ for $r^{c}=2^{1 / 6} \sigma$ ).

\begin{tabular}{clcc}
\hline No. & \multicolumn{1}{c}{$\lambda$} & $D^{\text {ar }}$ & $D^{\text {r }}$ \\
\hline 1 & $2 \cdot 010^{-9}$ & - & 0.479 \\
2 & $2 \cdot 10^{-6}$ & - & 0.216 \\
3 & 0.0002 & 0.133 & 0.095 \\
4 & 0.02 & 0.053 & 0.051 \\
5 & 0.20 & 0.039 & - \\
6 & 0.60 & 0.036 & 0.044 \\
7 & 1.00 & 0.039 & 0.033 \\
8 & 1.40 & 0.037 & 0.034 \\
\hline
\end{tabular}

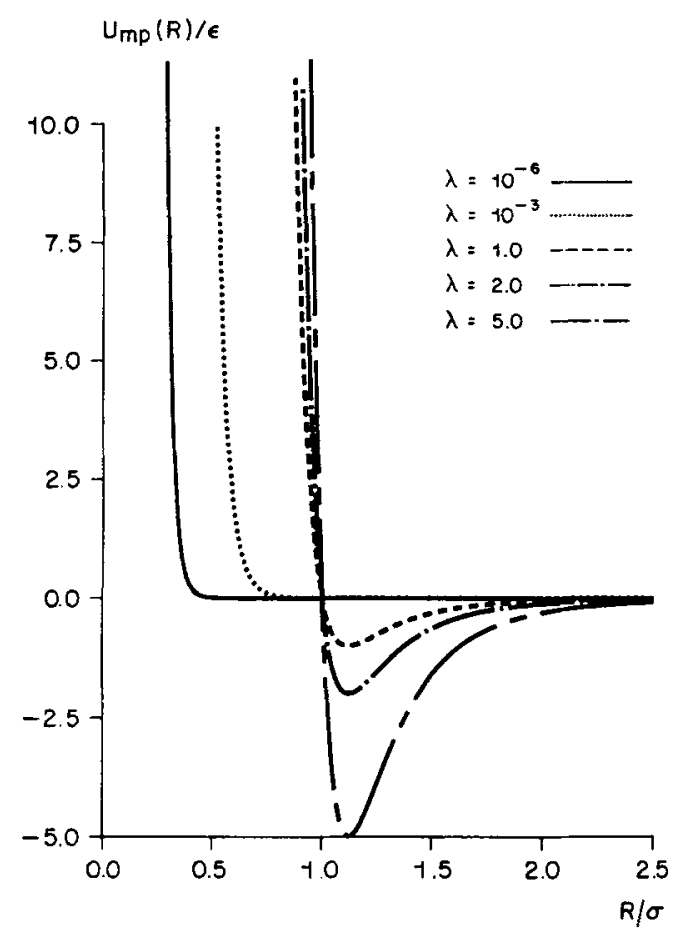

Figure 3. The Lennard-Jones potential for several values of the coupling parameter $\lambda$. 


\subsection{Centre-of-mass diffusion coefficients}

The centre-of-mass diffusion coefficients were obtained from the centre-of-mass displacement and from the centre-of-mass velocity autocorrelation function. We found that both methods gave consistent results, which are given in table 3 .

The results demonstrate that the diffusion coefficient is significantly influenced by the quality of the solvent. In figure 3 we have plotted the Lennard-Jones potential for several values of $\lambda$. Detailed inspection of this figure shows that the effective diameter of the monomers and polymer segments increases as $\lambda$ is increased. Therefore, the movement of the polymer will be hindered by the monomers as $\lambda$ increases. This causes the decrease of the diffusion coefficient. Comparison of the results as given in table 3 demonstrates that inclusion of the attractive part of the potential has only a minor influence on the diffusion coefficient.

\subsection{Radial distribution functions and Helmholtz free energy}

In figures 4 and 5 we have plotted the monomer-monomer $\left(g_{\mathrm{mm}}(r)\right)$ and monomer-polymer segment $\left(g_{\mathrm{mp}}(r)\right)$ radial distribution functions for several values of $\lambda$.

We did not observe any influence of $\lambda$ on $g_{\mathrm{mm}}(r)$. However, the behaviour of $g_{\mathrm{mp}}(r)$ as a function of $\lambda$ is interesting. From the intermolecular potential (cf. figure 3) we see that for low values of lambda the attractive part of the potential has become very small and the monomers and polymer segments can approach each other more closely. At high values of $\lambda$ the distance of approach does not change very much, but the attractive part of the potential becomes more important.

This behaviour of the potential as a function of lambda is reflected in the behaviour of $g_{\mathrm{mp}}(r)$. At low values of $\lambda$ the first peak is shifted considerably towards the left with respect to $g_{\mathrm{mm}}(r)$. At high values of lambda the first peak occurs almost for the same value of $r$ but increases in height. Note that due to the hinderance of a polymer segment by its neighbours, the first peak of the $g_{\mathrm{mp}}(r)$ is always lower than the first peak of $g_{\mathrm{mm}}(r)$ for the values of $\lambda$ we have considered. It is interesting to note that for very low values of $\lambda$ the effective size of a polymer segment as seen by a

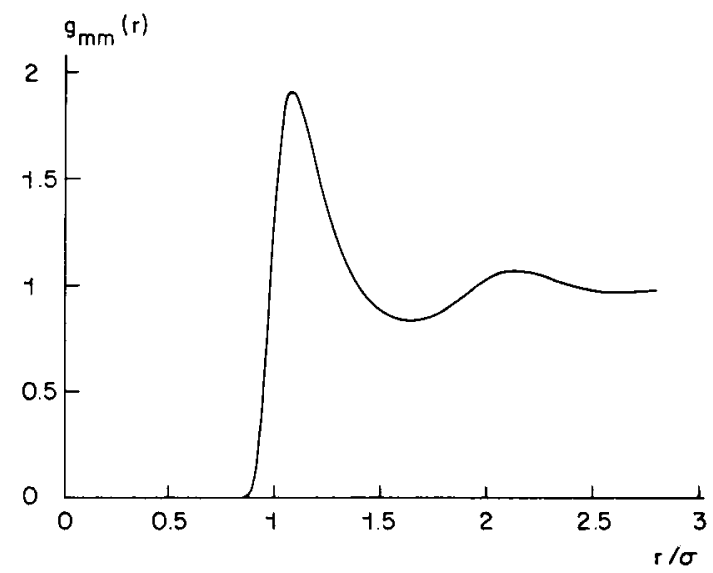

Figure 4. The monomer-monomer radial distribution function. Note that no influence of $\lambda$ on this function could be observed. 


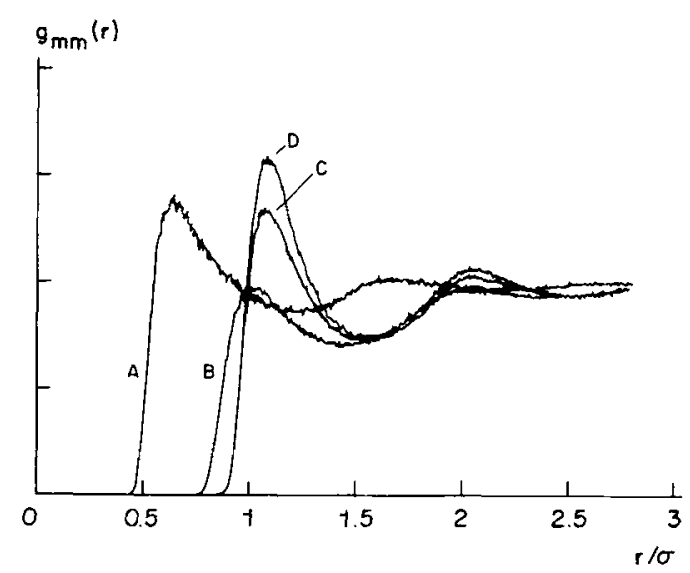

Figure 5. The monomer-polymer segment radial distribution function for various values of $\lambda:(A) \lambda=2 \times 10^{-4},(B) \lambda=2 \times 10^{-1},(C) \lambda=1 \cdot 0$, and $(D) \lambda=1 \cdot 4$.

monomer of the solvent, has become so small that a monomer can pass between two neighbouring polymer segments. Therefore, the spherical hindrance by the neighbours of the polymers is becoming less important. This is reflected in increased height of the first peak for these low values of $\lambda$.

For small values of $\lambda$ the $g_{\mathrm{mp}}(r)$ can be approximated by

$$
g_{\mathrm{mp}}(r) \approx \exp \left(-\lambda U^{\circ}(r) / k T\right)
$$

and for $\lambda=0$ there are no interactions between the polymer segments and the monomers. The polymer segments behave completely independently and the radial distribution function becomes:

$$
g_{\mathrm{mp}}(r)=1 .
$$

It is interesting to note that by studying the effect of the quality of the solvent the free energy of this system can be obtained. In our system the total potential energy can be written as

$$
U=U_{\mathrm{mm}}+U_{\mathrm{mp}}+U_{\mathrm{pp}}+U^{\text {harm }}
$$

where $U_{\mathrm{mm}}$ is the contribution of the monomer-monomer interactions, $U_{\mathrm{mp}}$ of the monomer-polymer segment interactions, $U_{\mathrm{pp}}$ of the polymer segment-polymer segment interactions and $U^{\text {harm }}$ the contribution of the harmonic potential.

In the expression for $U_{\mathrm{mp}}$ we have introduced the parameter $\lambda$ as a measure of the quality of the solvent. This parameter can be regarded as a coupling parameter, and when $\lambda$ is increased from 0 to $\lambda^{\prime}$ the change of the Helmholtz free energy can be obtained using Kirkwood's coupling parameter formalism [14]

$$
A_{\mathrm{c}}\left(\lambda^{\prime}\right)-A_{\mathrm{c}}(0)=\int_{0}^{\lambda^{\prime}} d \lambda\left\langle\left(\partial U_{\mathrm{mp}} / \partial \lambda\right)\right\rangle,
$$

with

$$
\left\langle\partial U_{\mathrm{mp}} / \partial \lambda\right\rangle=4 \pi \rho N_{\mathrm{m}} \int_{0}^{R^{\mathrm{c}}} d r r^{2} g_{\mathrm{mp}}(r, \lambda) U^{0}(r)
$$


where

$$
U^{0}(r)=4 \varepsilon^{0}\left[(\sigma / r)^{12}-(\sigma / r)^{6}\right]
$$

and $g_{\mathrm{mp}}(r, \lambda)$, the radial distribution function of the monomers and polymer segments, is dependent on $\lambda . N_{\mathrm{m}}$ is the number of monomers.

Substitution of equation (4) into the expression for $\left\langle\partial U_{\mathrm{mp}} / \partial \lambda\right\rangle$ (7) creates a divergence. Note that for small values of $r$ only the repulsive part of the potential $\left(1 / r^{12}\right)$ contributes to the integral in equation (7) and that the average energy of a particle interacting with a fixed source of energy $1 / r^{12}$ is of the order of $\lambda^{-3 / 4}[16]$. Therefore the integral over $\lambda$ does exist, although the divergence of $\left\langle\partial U_{\mathrm{mp}} / \partial \lambda\right\rangle$ can cause numerical difficulties, as pointed out by Swope et al. [17] and Shing et al. [18].

In figure 6 we have plotted $\ln \left(\left\langle\partial U_{\mathrm{mp}} / \partial \lambda\right\rangle\right)$ as a function of $\ln \lambda$. For very low values of $\lambda$ the values of $\ln \left(\left\langle\partial U_{\mathrm{mp}} / \partial \lambda\right\rangle\right)$ lie approximately on a straight line

$$
\ln \left(\left\langle\partial U_{\mathrm{mp}} / \partial \lambda\right\rangle\right)=A \ln (\lambda)+B
$$

with $A=0.73$ and $B=2 \cdot 93$.

We have solved the numerical difficulties by splitting the integral for the Helmholtz free energy into two parts by introducing $\lambda^{*}$

$$
A_{\mathrm{c}}\left(\lambda^{\prime}=1\right)-A_{\mathrm{c}}(0)=\int_{0}^{\lambda^{*}} d \lambda\left\langle\left(\partial U_{\mathrm{mp}} / \partial \lambda\right)\right\rangle+\int_{\lambda^{*}}^{\lambda^{\prime}} d \lambda\left\langle\left(\partial U_{\mathrm{mp}} / \partial \lambda\right)\right\rangle .
$$

The first integral has been calculated by substitution of the approximation, equation (9), which is valid for very small values of $\lambda$. The second integral is calculated directly from the radial distribution functions.

In table 4 we give the values for the Helmholtz free energy for several choices of $\lambda^{*}$. For small values of $\lambda^{*}$ the second integral becomes unreliable, due to numerical

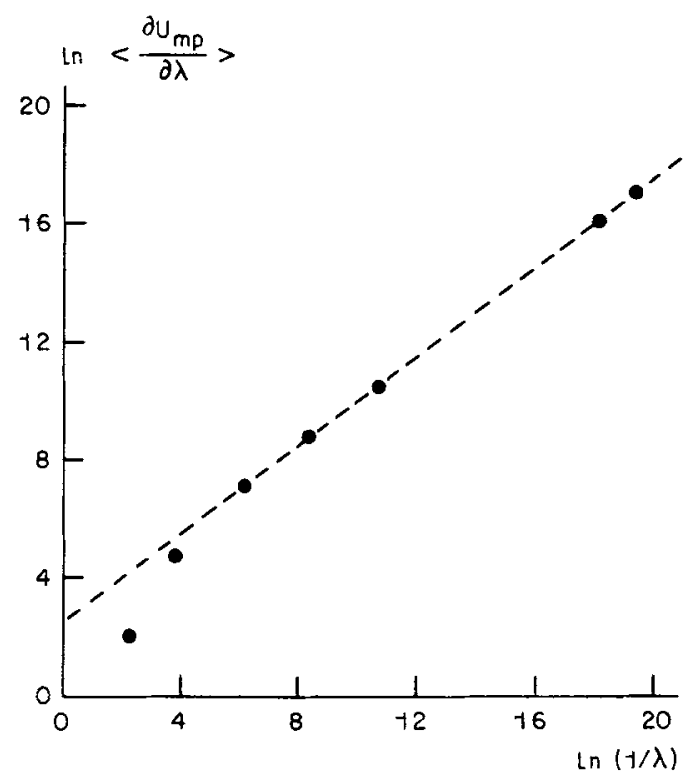

Figure 6. Approximation of $\left\langle\partial U_{\mathrm{mp}} / \partial \lambda\right\rangle$ as a function of $\ln (1 / \lambda)$ by a straight line for very small values of $\lambda$. 
Table 4. Calculation of the Helmholtz free energy difference. $I_{a}$ was calculated with equation (11) and $I_{b}$ was obtained directly from the simulations.

\begin{tabular}{|c|c|c|c|c|}
\hline No. & $\lambda$ & $I_{a}$ & $I_{b}$ & $\Delta A=I_{a}+I_{b}$ \\
\hline 1 & $1 \cdot 1210^{-8}$ & 0.37 & $2 \cdot 47$ & $2 \cdot 84$ \\
\hline 2 & $2.0010^{-5}$ & $2 \cdot 70$ & 0.97 & 3.67 \\
\hline 3 & $1.4610^{-4}$ & $4 \cdot 59$ & -0.94 & 3.65 \\
\hline 4 & $2.0010^{-4}$ & $5 \cdot 00$ & -1.54 & $3 \cdot 55$ \\
\hline 5 & $2.0010^{-3}$ & $9 \cdot 24$ & -5.63 & $3 \cdot 61$ \\
\hline 6 & $2.0010^{-2}$ & $17 \cdot 09$ & -11.13 & $5 \cdot 96$ \\
\hline
\end{tabular}

difficulties, for high values of $\lambda^{*}$ the first integral becomes unreliable, as can be seen from figure 6 . In between, we find that the Helmholtz free energy difference is independent of $\lambda^{*}$.

By studying the influence of the quality of the solvent we were able to calculate the difference in free energy between a system in which a polymer is immersed in a solvent of its own segments and a system in which the polymer and solvent do not have any interactions. This Helmholtz free energy difference is closely related to the excess Helmholtz free energy (see Appendix A), which is commonly used for estimating the phase behaviour of systems.

\section{Polymer in a sack model}

In this section we will present a simple model which can calculate the behaviour of the radius of gyration of a polymer immersed in a solvent as a function of the quality of this solvent.

\subsection{Introduction}

A qualitative explanation of the behaviour of the radius of gyration as a function of the quality of the solvent can be given if we consider figure 3 . In this figure the Lennard-Jones potential is given for several values of $\lambda$. It can be seen that at very low values of $\lambda$ the attractive part of the potential is very small. Therefore, the interactions between the polymer segments and the monomers of the solvent is mainly repulsive. So, when $\lambda$ is turned on, the polymer segments are 'suddenly' aware of these repulsive monomers. From figure 1 it can be seen that these 'repulsive' monomers cause the chain to 'collapse'. At higher values of $\lambda$ the attractive part of the potential starts to dominate. When $\lambda$ becomes greater than one, it is energetically favourable for the monomers to be in the neighbourhood of the polymer. This energy gain can compensate for the loss of entropy caused by a stretching of the chain and causes an increase of the radius of gyration.

\subsection{Description of a polymer in a sack model}

It is a formidable task to calculate the configuration of a polymer using rigorous statistical mechanical methods, especially if one has to take into account the influence of the solvent. We have developed a simple method to approximate the chain statistics so that the influence of the solvent can be studied using classical thermodynamics. 


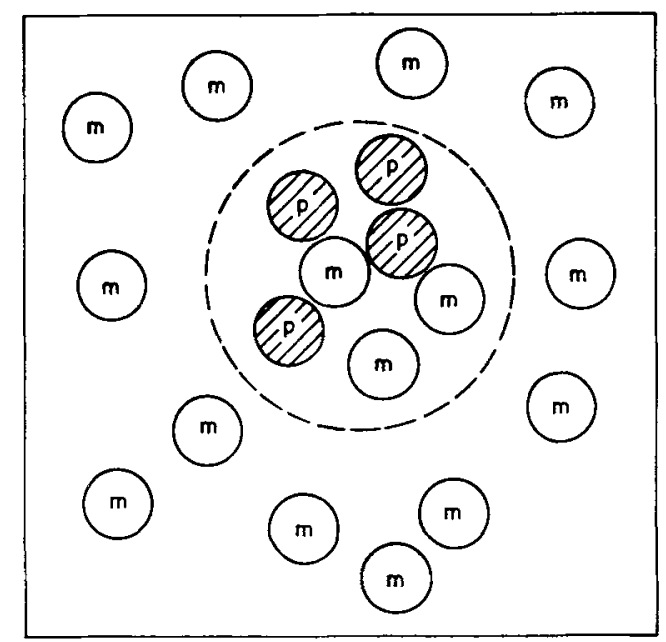

Figure 7. Schematic drawing of the polymer in a sack model.

In the model we have used in the MD simulations, the polymer segments differ from the monomers by the springs which connect the segments into a chain. These springs restrict the configurations of the polymer. When one of the polymer segments is localized at a certain place the other segments can only be in a certain volume around this particle and the polymer segments are not randomly distributed in this volume but each segment is linked to its neighbours.

In our model we have disregarded this last restriction on the configurations of the polymer segments. We assume that the polymer segments are distributed randomly inside a sphere and can be treated as independent particles. The volume of the sphere is variable and is a measure of the radius of gyration (see Appendix B). This sphere is immersed in an infinite bath of monomers. The membrane of the sphere is semi-permeable: the polymer segments cannot pass the membrane and are therefore restricted to move inside the sphere. The monomers are allowed to pass the membrane freely (figure 7 ).

With this model the problem of calculating the radius of gyration is replaced by a phase equilibrium problem. The semi-permeable sphere has to be in equilibrium (mechanical and chemical) with the bath of monomers. The equilibrium conditions for this system are as follows: the fugacity of the monomers in the sphere has to be equal to the fugacity of the monomers outside the sphere (chemical equilibrium), the pressure outside the sphere has to be equal to the pressure inside the sphere (mechanical equilibrium). These equilibrium conditions can be stated as

$$
\begin{aligned}
& f_{\mathrm{m}}^{\mathrm{s}}=f_{\mathrm{m}}^{\mathrm{B}}, \\
& \mathbf{P}^{\mathrm{in}}=\mathbf{P}^{\text {out}},
\end{aligned}
$$

where $f_{\mathrm{m}}$ is fugacity of the monomers in the bulk $(B)$ or in the sphere (S) and $\mathrm{P}$ is pressure in(side) or out(side) the sphere. The fugacity of a compound can be obtained from an equation of state using:

$$
R T \ln \left(f_{i} / x_{i} P\right)=\left[\int_{V}\left[\left(\partial P / \partial N_{i}\right)_{T, V, N_{j}}-R T / V\right] d V\right]-R T \ln (V P / N R T) .
$$


The pressure and the fugacity are calculated from a new equation of state based on the van der Waals equation. A detailed discussion on the development of this equation is published elsewhere $[19,20]$. In this paper we will postulate this equation and we will show that it can qualitatively describe the behaviour of the mixture of polymer segments and monomers for various values of $\lambda$.

\subsection{Equation of state}

Before we postulate this new equation of state we will first demonstrate why the van der Waals equation of state with the conventional mixing rules fails to describe the behaviour of this mixture of monomers and polymer segments.

If we consider the Lennard-Jones potential which describes the interaction of the monomers (m-particle) with the polymer segments (p-particle) as a function of $\lambda$ the following limiting behaviour can be observed. For $\lambda=1$ there is no difference in interaction of the polymer segments with the monomers compared to the $\mathrm{m}-\mathrm{m}$ interaction or to the $\mathrm{p}-\mathrm{p}$ interaction. In this case the system behaves like a pure component. For $\lambda=0$ there is no interaction of the $\mathrm{m}$ particles with the particles, although the interactions $m-m$ and $p-p$ have not been changed. In this special case the $\mathrm{m}$ and $\mathrm{p}$ particles are completely independent and behave like pure components. If the value of lambda is increased the $p$ and $m$ particles become aware of each other.

Let us assume that the van der Waals equation can describe the pure components sufficiently accurately and focus our attention on the conventional mixing rules. For a pure component the van der Waals equation is

$$
p^{*} / T^{*}=N /\left(V^{*}-N b\right)-N^{2} a / T^{*} V^{* 2} \text {. }
$$

This equation can be used to describe the behaviour of a mixture with the following mixing rules:

$$
\begin{aligned}
a & =\sum_{i} \sum_{j} x_{i} x_{j} a_{i j}, \\
b & =\sum_{i} x_{i} b_{i}
\end{aligned}
$$

and the combining rule

$$
a_{i j}=\left(1-k_{i j}\right)\left(a_{i i} a_{j j}\right)^{1 / 2}
$$

in which a binary interaction coefficient $k_{i j}$ is introduced.

In the limiting case $\lambda=0$ there is no interaction between the monomers and polymer segments, so for the energy parameter in the van der Waals equation it is reasonable to assume

$$
a_{\mathrm{mp}}=0 \text {. }
$$

For this binary $\mathrm{m}-\mathrm{p}$ mixture and for $\lambda=0$ the van der Waals equation is

$$
\begin{aligned}
p^{*} / T^{*}= & N_{\mathrm{p}} /\left(V^{*}-N_{\mathrm{p}} b_{\mathrm{p}}-N_{\mathrm{m}} b_{\mathrm{m}}\right)-N_{\mathrm{p}}^{2} a_{\mathrm{pp}} / T^{*} V^{* 2} \\
& +N_{\mathrm{m}} /\left(V^{*}-N_{\mathrm{p}} b_{\mathrm{p}}-N_{\mathrm{m}} b_{\mathrm{m}}\right)-N_{\mathrm{m}}^{2} a_{\mathrm{mm}} / T^{*} V^{* 2} .
\end{aligned}
$$

This equation is inconsistent with the picture in the $\lambda=0$ limit. In this limit, the two components are non-interacting and should make independent contributions to the pressure. In the first term on the right the accessible volume of the p-particles is 
given as the total volume of the system $(V)$ minus the sum of the 'hard core'excluded volume of the p-particles $\left(N_{\mathrm{p}} b_{\mathrm{p}}\right)$ and the 'hard core'-excluded volume of the m-particles $\left(N_{\mathrm{m}} b_{\mathrm{m}}\right)$. Although the particles are by definition non-interacting, the van der Waals equation predicts an accessible volume which depends on the other species for this limit. The reason for this incorrect behaviour in this limit becomes clear if we realize that the derivation of the mixing rule for the $b$ parameter is based on a hard sphere model [21]. In such a model the distance at which a particle can approach another particle is independent of binary interactions. However, in our monomer polymer segment mixture the effective diameter of the particles is dependent on $\lambda$, as can be seen from the intermolecular potential (see figure 3 ).

This inconsistency in this limit of the van der Waals equation with the hard sphere mixing rules can be resolved if we adopt the concept of apparent size as probed by a particular molecule [19, 20]. This concept states that the accessible volume of a particle $i$ is the total volume minus the total apparent volume of the particles $j$ as probed by particle $i$.

In the system we are considering the apparent size of a particle for an $\mathrm{m}$ particle is dependent on $\lambda$, while the apparent size of an $\mathrm{m}$ particle for another $\mathrm{m}$ particle is independent of $\lambda$. This concept of apparent size in the van der Waals equation for this system gives

$$
\begin{aligned}
p^{*} / T^{*}= & N_{\mathrm{p}} /\left(V^{*}-N_{\mathrm{p}} b_{\mathrm{p}}-N_{\mathrm{m}} b_{\mathrm{pm}}\right)-N_{\mathrm{p}}^{2} a_{\mathrm{pp}} / T^{*} V^{* 2}+-2 N_{\mathrm{p}} N_{\mathrm{m}} a_{\mathrm{mp}} / T^{*} V^{* 2} \\
& +N_{\mathrm{m}} /\left(V^{*}-N_{\mathrm{p}} b_{\mathrm{mp}}-N_{\mathrm{m}} b_{\mathrm{m}}\right)-N_{\mathrm{m}}^{2} a_{\mathrm{mm}} / T^{*} V^{* 2}
\end{aligned}
$$

where $b_{\mathrm{pm}}$ is the apparent volume of an m-particle for a p-particle, and $b_{\mathrm{mp}}$ is the apparent volume of a p-particle for an m-particle.

Inspection of this equation shows that in the limit $\lambda=0$, where

$$
a_{\mathrm{mp}}=0 \quad \text { and } \quad b_{\mathrm{mp}}=b_{\mathrm{pm}}=0
$$

the total pressure is indeed the sum of the two independent contributions of the monomers and polymer segments.

\subsection{Estimation of the parameters of the equation-of-state}

In order to apply the equation of state to the phase equilibrium problem we have to estimate the $a$ and $b$ parameters for the monomers and polymer segments. The monomers and polymer segments are both (identical) pure Lennard-Jones fluids and the values for the $a$ and $b$ parameters can in principle be calculated from various properties of a pure Lennard-Jones fluid such as the critical data or a fit to the equation of state proposed by Nicolas et al. [21]. However, they will all give different sets of values for these parameters because the van der Waals equation is too 'simple' to accurately describe the behaviour of a pure Lennard-Jones fluid. We have used the critical data to estimate the $a$ and $b$ parameters for our calculations (see table 5).

We emphasize that we are mainly interested in a qualitative explanation of the behaviour of the radius of gyration and therefore we have made no attempt to optimize the parameters. In all expressions we have tried to use the simplest equations which qualitatively match the behaviour of the monomer segment system.

In the conformal solution theory the parameters from the equation of state are related to the intermolecular potential. From this theory an expression for $a_{\mathrm{pm}}$ and 
Table 5. Properties of the pure Lennard-Jones fluid (Nicolas et al., [22]), $T_{\mathrm{c}}^{*}$ : critical temperature, $P_{c}^{*}$ : critical pressure and $\rho_{c}^{*}$ : critical volume (all reduced units). $a$ and $b$ are the parameters of the van der Waals equation (note that $\rho_{\mathrm{c}}^{*}=1 / 3 b$ and $P_{\mathrm{c}}^{*}=a / 27 b^{2}$ ).

\begin{tabular}{cl}
\hline$T_{\mathrm{c}}^{*}=1.35$ & $(k T / \varepsilon)$ \\
$P_{\mathrm{c}}^{*}=0.142$ & $\left(p \sigma^{3} / \varepsilon\right)$ \\
$\rho_{\mathrm{c}}^{*}=0.35$ & $\left(\rho / \sigma^{-3}\right)$ \\
$b=10.95$ & $\left(\sigma^{3}\right)$ \\
$a=3.48$ & $\left(\sigma^{3} \varepsilon\right)$ \\
\hline
\end{tabular}

$b_{\mathrm{pm}}$ can be obtained [22]

$$
\begin{aligned}
& a_{\mathrm{pm}}=a\left(\varepsilon_{\mathrm{pm}} / \varepsilon_{\mathrm{mm}}\right)\left(d_{\mathrm{pm}} / d_{\mathrm{mm}}\right)^{3}=\lambda a\left(d_{\mathrm{pm}} / d_{\mathrm{mm}}\right)^{3}, \\
& b_{\mathrm{pm}}=b\left(d_{\mathrm{pm}} / d_{\mathrm{mm}}\right)^{3},
\end{aligned}
$$

where $\varepsilon_{i j}$ is the characteristic energy parameter of the intermolecular potential, $d_{i j}$ is the effective hard sphere diameter, and $a=a_{\mathrm{mm}}, b=b_{\mathrm{mm}}$ are the parameters of the equation of state for the pure Lennard-Jones fluid.

For a given potential Barker and Henderson [22] defined an effective hardsphere diameter $d_{i j}$ as

$$
d_{i j}=\int_{0}^{\sigma}\left\{1-\exp \left[-\beta u_{i j}(r)\right]\right\} d r
$$

where $\sigma$ is the value of $r$ for which $u_{i j}(r)$ is zero, $\beta$ is $1 / T^{*}$ and $i, j$ denotes mm, mp or $\mathrm{pp}$ in this case. Note that with these definitions $a_{\mathrm{mp}}$ and $b_{\mathrm{mp}}$ both go to zero if the intermolecular potential which describes the $m-p$ interaction vanishes.

We have assumed that the sphere is immersed in an infinite bath of monomers. The fugacity and pressure outside the sphere are independent of the polymer segments and therefore independent of $\lambda$. The fugacity of the monomers outside the sphere follows directly from the equation of state. The pressure outside the sphere, which corresponds to the pressure of the bath of monomers, is calculated indirectly by substitution of one 'experimental value' of the radius of gyration (we have used the value for $\lambda=1$ ). The equilibrium conditions (11) and (12), with (19) for the pressure are solved numerically using a two dimensional Newton-Raphson method to calculate the number of monomers inside the sphere $\left(N_{\mathrm{m}}\right)$ and the volume of the sphere $\left(V_{\mathrm{s}}\right)$.

\subsection{Results}

In figure 1 we present the results of the calculations with the polymer in a sack model. The calculations predict a decrease of the radius of gyration of the polymer with the solvent is turned on. A further increase of $\lambda$ causes the radius of gyration to increase again.

Comparison of these calculations with the data obtained from the simulations shows a remarkable agreement. Only at very low values of $\lambda$ do the calculations deviate from the simulation data.

We have also done some calculations for the case in which only the repulsive part of the Lennard-Jones potential is taken into account. For this case, in which there are no attractive forces, we have assumed $a=a_{\mathrm{mm}}=a_{\mathrm{pp}}=a_{\mathrm{mp}}=0$ and for 
convenience we have used the same value for $b$ as in the previous calculations. Because there are no attractive forces between the polymer segments one would not expect a 'collapse' of the polymer as the solvent is introduced. This disappearance of the sudden decrease of the radius of gyration is observed both in the simulations and for the polymer in a sack model. The latter calculations predict a slight decrease of the radius of gyration as $\lambda$ increases (cf. figure 2). Although the results from the simulations show a similar trend, this decrease could not be confirmed rigorously, owing to the inaccuracy of these data.

\section{Conclusions}

In this paper we have introduced the concept of quality of the solvent, as a rough approximation of the chemical nature of the solvent. It turns out that varying this quality has a significant influence on the dynamic and static properties of a polymer. Furthermore, this approach enables us to calculate the Helmholtz free energy, which is important for calculating phase behaviour. Numerical results for the diffusion coefficient and the radius of gyration have been obtained from molecular dynamics calculations. The latter showed an unexpected, non-monotonic, behaviour.

The behaviour of the diffusion coefficient, as a function of the quality of the solvent, can be understood from the effective size of the solvent particles which is determined by the repulsive part of the potential. The attractive part of the potential has only minor influence on the diffusion. On the other hand, for the behaviour of the radius of gyration both the repulsive and the attractive part of the potential turn out to be important.

We have presented a simple model which turns out to be very useful, in spite of the complexity of this kind of problem. The introduction of some assumptions for handling the chain statistics in this model reduces the calculations to a classical phase equilibrium problem which, despite its simplicity, leads to a fair description of the observed behaviour.

The authors wish to acknowledge the contributions of M. A. J. Michels.

\section{Appendix A}

\section{Helmholtz free energy of mixing}

For estimating the phase behaviour one is interested in the Helmholtz free energy of mixing

$$
\Delta A_{\text {mix }}=A_{\text {(actual solution) }}-A_{\text {(pure components) }} .
$$

In figure 8 we have given a thermodynamic cycle for a binary mixture. In this cycle we can distinguish the following steps:

(0) mixing of two components $\left(\Delta A_{\text {mix }}\right)$;

(1) expansion of the pure components $\left(\Delta_{1} A\right)$;

(2) mixing of the two components with no interaction between the two components $\left(U_{12}=0, \Delta A_{2}=0\right)$;

(3) increase of the interaction from $U_{12}=0$ to the actual value in the mixture $\left(\Delta A_{1}\right)$. 


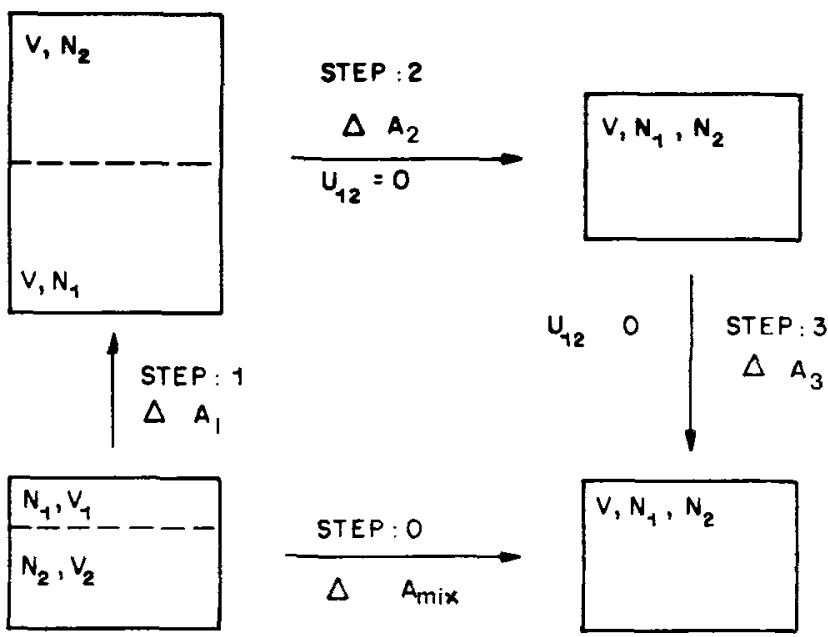

Figure 8. Thermodynamic cycle for calculating the Helmholtz free energy of mixing.

The Helmholtz free energy of mixing can thus be written as

$$
\Delta A_{\text {mix }}=\Delta A_{1}+\Delta A_{2}+\Delta A_{3} \text {. }
$$

The difference in Helmholtz free energy of step (3) in the cycle is just the free energy we have obtained from the molecular dynamics simulations:

$$
\Delta A_{\text {sim }}=\Delta A_{\text {mix }}-\Delta A_{1} \text {. }
$$

From this equation we see that the Helmholtz free energy, as obtained by the computer simulations, is closely related to the important Helmholtz free energy of mixing. Note that $\Delta A_{1}$ involves only pure components and therefore contains no information about the mixing process.

\section{Appendix B}

Relation between the volume of the sphere and radius of gyration

In the model we have used, it is assumed that the polymer segments are distributed randomly inside the sphere. If a time average is taken there will be a uniform distribution of polymer segments inside the sphere.

The radius of gyration $(S)$ of a molecule is defined as the distance from the centre-of-mass at which a point mass, with the same mass as the molecule, has to be in order to have the same moment of inertia

$$
M S^{2}=\int_{m} r^{2} d m
$$

For a uniform distribution of polymer segments

$$
d m=\rho 4 \pi r^{2} d r .
$$

and

$$
S^{2}=\int_{0}^{R} r^{2} \rho 4 \pi r^{2} d r / \int_{0}^{R} \rho 4 \pi r^{2} d r=(3 / 5) R^{2}
$$


where $R$ is the radius of the sphere which contains the polymer segments.

The relation between the volume of the sphere $\left(V_{s}\right)$ and the radius of gyration $(S)$ is:

$$
S^{2}=(3 / 5)\left(3 V_{\mathrm{s}} / 4 \pi\right)^{2 / 3}
$$

\section{References}

[1] Webman, I., Lebowitz, J. L., and Kalos, M. H., 1981, Macromolecules, 14, 1495.

[2] Bishop, M., and Michels, J. P. J., 1986, J. chem. Phys., 84, 447.

[3] Bruns, W., and Bansal, R., 1981, J. chem. Phys., 74, 2064.

[4] BRuns, W., and BANSAL, R., 1981, J. chem. Phys., 75, 5149.

[5] Bishop, M., Kalos, M. H., and Frisch, H. L., 1983, J. chem. Phys., 79, 3500.

[6] RAPAPORT, D. C., 1979, J. Phys. A, 71, L231.

[7] Bishop, M., Kalos, M. H., and Frisch, H. L., 1979, J. chem. Phys., 70, 1299.

[8] Frisch, H. L., Bishop, M., CePerley, D., and Kalos, M. H., 1980, J. Macromolecules Sci. Phys. B, 18, 453.

[9] Rapaport, D. C., 1979, J. chem. Phys., 71, 3299.

[10] Khalatur, P. G., Papulov, Yu. G., and Pavlov, A. S., 1986, Molec. Phys., 58, 887.

[11] Smit, B., Van der Put, A., Peters, C. J., Swahn Arons, J. De, and Michels, J. P. J., 1988, J. chem. Phys., 88, 3372.

[12] Toxvaerd, S., 1987, J. chem. Phys., 86, 3667.

[13] Smit, B., Van der Put, A., Peters, C. J., Swaan Arons, J. De, and Michels, J. P. J., 1988, Chem. Phys. Lett., 144, 555.

[14] HaIle, J. M., 1986, Fluid Phase Equilib., 26, 103.

[15] Verlet, L., 1967, Phys. Rev., 159, 98.

[16] Squire, D. R., and Hoover, W. G., 1969, J. chem. Phys., 50, 701.

[17] Swope, W. C., and ANDerson, H. C., 1984, J. phys. Chem., 88, 6548.

[18] Shing, K. S., and Chung, S. T., 1987, J. phys. Chem., 91, 1674.

[19] Smit, B., and Cox, K. R., 1988, Fluid Phase Equilib., 43, 171.

[20] Smit, B., and Cox, K. R., 1988, Fluid Phase Equilib., 43, 181.

[21] Mansoori, G. A., 1986, Equations of State, edited by K. C. Chao and R. L. Robinson (ASC Symp. Ser. 300, American Chemical Society, Washington, D.C.), Chap. 15.

[22] Nicolas, J. J., Gubbins, K. E., Street, W. B., and Tildesley, D. J., 1979, Molec. Phys., $37,1429$.

[23] Barker, J. A., and Henderson, D., 1967, J. chem. Phys., 47, 4714. 